\title{
Different Classes of Glutamate Receptors and GABA Mediate Distinct Modulations of a Neuronal Oscillator, the Medullary Pacemaker of a Gymnotiform Electric Fish
}

\author{
Masashi Kawasaki ${ }^{a}$ and Walter Heiligenberg \\ Neurobiology Unit, Scripps Institution of Oceanography, University of California at San Diego, La Jolla, California 92093
}

\begin{abstract}
Gymnotiform electric fish generate distinct communicatory signals by modulating the rate of their electric organ discharges (EODs). Each EOD is triggered by a command pulse from the medullary pacemaker nucleus (PN), which contains pacemaker cells and relay cells. The firing rate of this nucleus is modulated by inputs from the diencephalic prepacemaker nucleus (PPN). The NMDA receptor blocker APV and the kainate/quisqualate receptor blocker CNQX, administered to the PN, suppress different types of modulations, indicating that different classes of glutamate receptors mediate the generation of different modulations. A comparison of the $\mathbf{2}$ genera, Hypopomus and Eigenmannia, reveals that sustained modulations, such as smooth rises in the rate of pacemaker cell firing and the selective silencing of the relay cells (only observed in Hypopomus), are mediated by NMDA receptors, whereas the brief and rapid acceleration, called "chirp" or "decrement burst," is mediated by kainate/quisqualate receptors.

Application of the GABA blocker bicuculline reveals that the $\mathbf{2}$ genera differ in the mechanism by which they slow the firing rate of their pacemaker. Whereas Hypopomus uses GABAergic inhibition to slow down and ultimately silence its pacemaker cells, Eigenmannia reduces tonic, APV-sensitive excitation originating from its PPN and lacks GABAergic inhibition in the PN.
\end{abstract}

Studies on pattern-generating neural systems have shown that specific transmitters and neural modulators are able to induce different modes of activities in anatomically fixed neuronal networks. Through control of such modulators, an animal can thus utilize the same neuronal hardware to generate qualitatively different behaviors (Marder, 1987; Harris-Warrick, 1988; Getting, 1989; Harris-Warrick and Marder, 1991). This notion has emerged mainly from studies of invertebrate systems, but it also holds for vertebrate systems: Gymnotiform electric fish provide convenient vertebrate preparations in which a variety of elec-

\footnotetext{
Received Apr. 4, 1990; revised July 20, 1990; accepted Aug. 10, 1990.

We thank John Dye for most helpful comments on this manuscript, Clifford H. Keller and Sally Keller for collecting Hypopomus in Venezuela, and Grace Kennedy for her expert technical assistance. This research was supported by NIMH Grant R37 MH 26149-14 and NINCDS Grant R01 NS 22244-04 to W.H.

Correspondence should be addressed to Walter Heiligenberg, Neurobiology Unit, Scripps Institution of Oceanography, University of California at San Diego, A-002, La Jolla, CA 92093. Reprint requests should be sent to Masashi Kawasaki, Department of Biology, Gilmer Hall, University of Virginia, Charlottesville, VA 22901.

a Present address: Department of Biology, Gilmer Hall, University of Virginia, Charlottesville, VA 22901.

Copyright (C) 1990 Society for Neuroscience $0270-6474 / 90 / 123896-09 \$ 03.00 / 0$
}

trical behaviors can be induced through modulations of a simple neuronal network in their pacemaker nucleus (PN).

A gymnotiform fish, Hypopomus, generates "pulse-type" electric organ discharges (EODs) at mostly regular frequencies between a few and several tens per second. While the wave form of the pulses is highly stereotyped, with a duration of a fcw milliseconds, the frequency of EOD pulses exhibits various modulations in different behavioral contexts (Westby, 1975; Hagedorn, 1983, 1986). The behavioral repertoire of Hypopomus brevirostris includes rises and falls of EOD frequency, 2 types of interruptions of EODs, and chirps (also referred to as "decrement bursts"; Hopkins and Heiligenberg, 1978; Heiligenberg and Bastian, 1980; Hopkins and Westby, 1986; Kawasaki and Heiligenberg, 1989). These modulations originate from the prepacemaker nucleus (PPN) in the diencephalon, which provides modulatory inputs to the PN in the medulla. Neurons in the pacemaker nucleus normally fire in synchrony, producing command pulses that trigger individual EOD pulses. Under the influence of modulatory inputs from the PPN, a simple neuronal circuit within the PN, which consists of only 2 types of neurons, operates in different modes and produces a variety of behavioral outputs (Kawasaki and Heiligenberg, 1989).

Similar, though less numerous, types of modulations are performed by the related genus, Eigenmannia, which, in contrast to Hypopomus, discharges its electric organ in a continual, wavetype manner. Recent neuropharmacological studies on the PN of wave-type genera have revealed that smooth rises in EOD frequency are mediated by NMDA receptors, whereas chirps are mediated by kainate/quisqualate receptor subtypes (Dye et al., 1989). This discovery encouraged us to explore the neuropharmacological basis of the richer repertoire of pacemaker modulations in Hypopomus.

In this study, we applied glutamate and its agonists, as well as blockers for different receptor subtypes, to the PN of $\mathrm{Hy}$ popomus and Eigenmannia, while various behaviors were induced either naturally or artificially by stimulating the PPN. We also applied GABA and its blocker bicuculline to test our earlier hypothesis that frequency falls are mediated by different mechanisms in pulse- and wave-type species.

\section{Materials and Methods}

We followed the methods applied in our earlier studies on Hypopomus (Kawasaki and Heiligenberg, 1989) and on Eigenmannia and Apteronotus (Dye et al., 1989). Animals were curarized by injection of 10-20 $\mu \mathrm{l}$ of a $2 \%$ Flaxedil solution in Ringer's solution and were gently suspended in a sponge-lined clamp with only the dorsal surface of their head protruding above the water line. They were respirated by a constant flow of aquarium water, approximately 2-4 drops per sec, through a tube inserted in their mouth. The residual EOD signal was recorded by a suction electrode placed over the tip of the tail. 

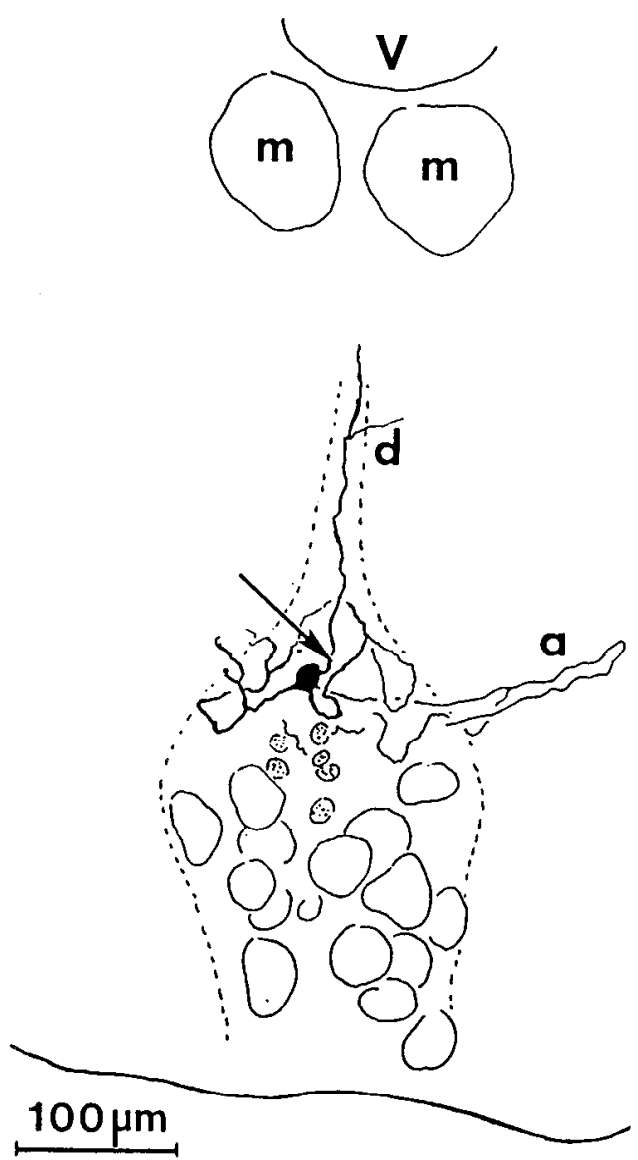

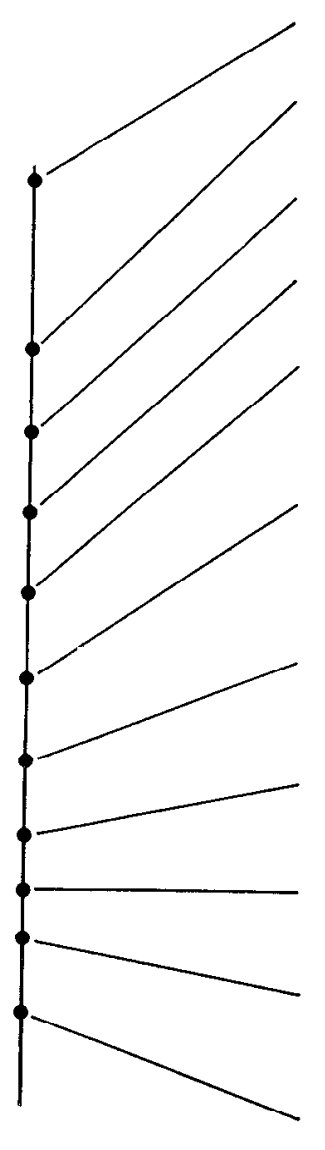

Field potential

Responses to glutamate $G A B A$

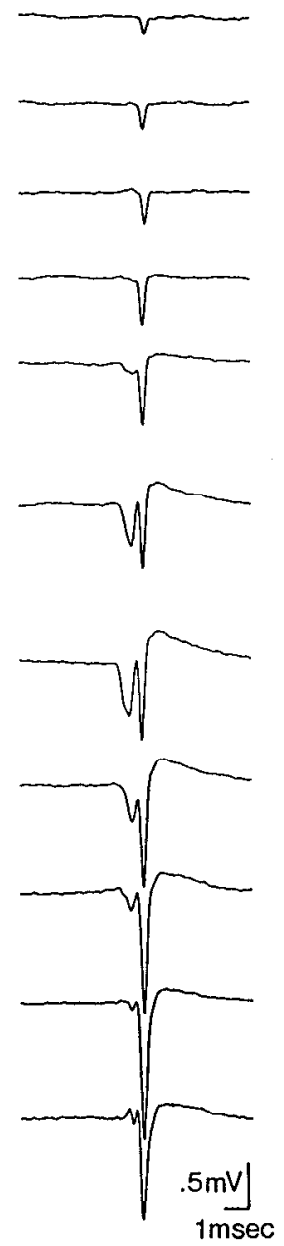

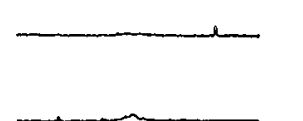
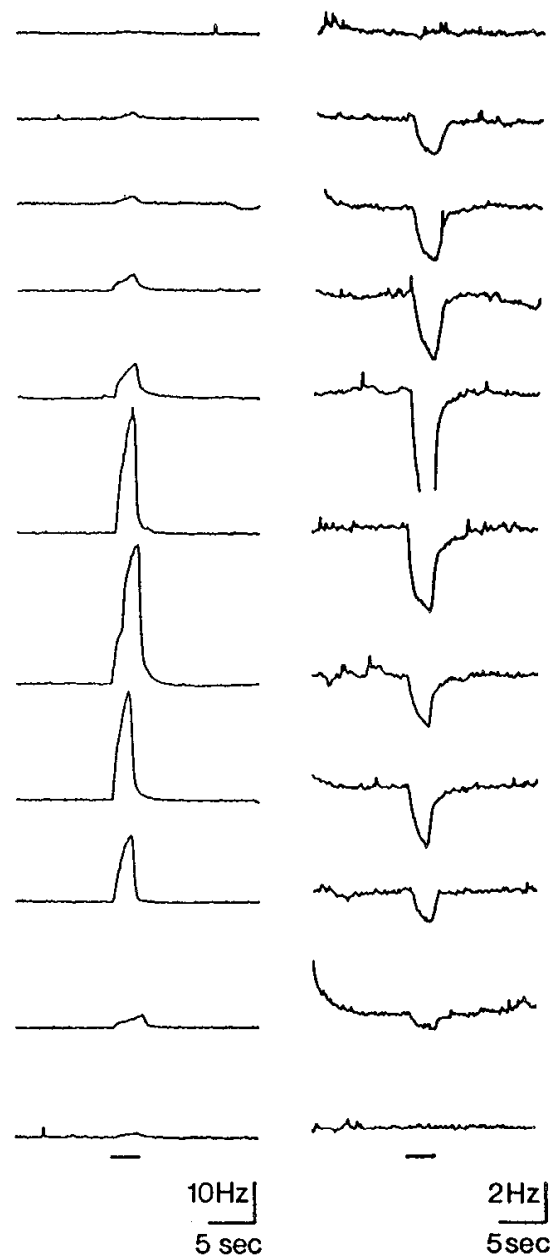

Figure 1. Left, Camera lucida drawing of the medullary PN of Hypopomus brevirostris, showing large relay cell somata ventrally and small pacemaker cell somata (stippled, one labeled black) dorsally. This pacemaker neuron was labeled by intracellular injection of HRP to reveal the structure of its dendrites $(d)$ and axon $(a)$. All labeled pacemaker cells showed the looped axon outside the nucleus. The PN rests on the ventral surface of the brain. $m$, Mauthner axons; $V$, ventricle. Right, Field potentials were recorded (left column), and iontophoretic effects of glutamate (center column) and GABA (right column) on EOD frequency were measured at different depths in the nucleus. Note that the first peak of the field potential is largest at the dorsal part of the nucleus, and the second peak is largest at the ventral part of the nucleus. The penetration line, which actually passed vertically through the center of the nucleus, indicates corresponding depths of recording and iontophoresis sites. These depths were determined in reference to the location of an alcian blue dye mark deposited at the tip of the arrow. This location corresponds to the sixth dot along the penetration line. As a result of a progressive frequency fall, the EOD was interruptcd in the fifth trace in the right column. By using the timing of EODs as a reference, field potential data were averaged 50 times to reduce noise. The resting frequency of the EOD was $11 \mathrm{~Hz}$.

After local application of Xylocaine, a small hole, approximately 1 $\mathrm{mm}$ in diameter, was drilled above a lateral boundary of the tectal commissure in order to reach the area of the PPN $1200-1600 \mu \mathrm{m}$ beneath. A small piece of cartilage connecting the parietal bones was excised above the caudal end of the cerebellum in order to reach the PN in the central medulla at a depth of $2200-2800 \mu \mathrm{m}$ from the cerebellar surface. A small area of a parietal bone was then glued with Histoacryl to a Plexiglas holder for mechanical stabilization.

The PN, which is unpaired, was penetrated with a glass capillary filled with $3 \mathrm{M} \mathrm{NaCl}$ for recording of its field potential. The exact location of the nucleus could be determined by the wave form of its potential (Fig. 1 , right). By means of a separate microdrive, the PPN, which is paired, was penetrated unilaterally with a glass capillary of a tip diameter of 5$10 \mu \mathrm{m}$ filled with $0.1 \mathrm{M}$ L-glutamate $(\mathrm{pH}, 8.0)$ for iontophoretic stimulation of its neurons. Depending upon the location of this stimulating electrode, each of 4 distinct types of pacemaker modulation could be elicited: frequency rises, frequency drops, chirps, or sudden interruptions (Kawasaki and Heiligenberg, 1989). After localization of the PN, the $\mathrm{NaCl}$ capillary was removed, and a triple-barrel electrode was advanced to the same location. One barrel was again filled with $3 \mathrm{M} \mathrm{NaCl}$ to record the field potential of the PN for accurate placement of the electrode, whereas the 2 remaining barrels were filled with solutions of glutamate, GABA, or their agonists, antagonists, or control solutions to be injected into the PN either by iontophoresis (glutamate, GABA, NMDA, kainate, and quisqualate) or by pressure (APV, CNQX, and bicuculline).

The pharmacological agents tested were glutamate (Sigma), GABA ( $\gamma$-amino- $n$-butyric acid, Sigma), the NMDA receptor blocker $\mathrm{D}(-) 2-$ amino-5-phosphonovaleric acid (APV; Cambridge Research Biochemicals), the kainate/quisqualate receptor blocker 6-cyano-7-nitroquinoxaline-2,3-dione (CNQX; Cambridge Research Biochemicals), the GABA blocker bicuculline methiodide (Sigma), the glutamate agonist NMDA ( $N$-methyl-D-aspartate, Sigma), kainic acid (Sigma), and quisqualic acid (Sigma). Although an initial batch of $\gamma$-D-glutamylaminomethylsulphonic acid (GAMS; Cambridge Research Biochemicals) had shown consistent effects when applied to the pacemakers of the wave-type 
species Eigenmannia and Apteronotus (Dye et al., 1989), subsequent batches of this drug from the same company failed to produce reliable effects in Eigenmannia and Hypopomus. The more recently developed kainate/quisqualate blocker CNQX yielded consistent effects in both species and, therefore, was employed in these experiments. All concentrations expressed in this report refer to the concentrations of agents in the capillary electrodes.

For pressure injection, we pushed a tightly fitting polyethylene tube into the open end of the barrel and connected the other end via a $T$ joint to a pressure line. By manually closing the open end of the $T$ joint, we could increase the pressure inside the barrel and force small quantities of solution out of the tip of the barrel (inner diameter, 5-10 $\mu \mathrm{m}$ ). On the basis of visual inspection of the size of a droplet extruded in air, we calculated that a quantity of approximately $0.1 \mathrm{nl}$ was ejected over a period of $1-5 \mathrm{sec}$. Even at threshold concentrations of the agent, this quantity was sufficient to cause noticeable effects when applied to the optimal location within the PN. An injection of this quantity of any solution, even an artificial cerebrospinal fluid (in mM: $124 \mathrm{NaCl}, 2 \mathrm{KCl}$, $1.25 \mathrm{KH}_{2} \mathrm{PO}_{4}, 1.1 \mathrm{MgSO}_{4}, 1.1 \mathrm{CaCl}_{2}, 16 \mathrm{NaHCO}_{3}$ ), which served as a control, could be detected by an immediate, brief rise of the pacemaker frequency, and auditory monitoring of this rise helped to limit the volume of the injection by manual control of the pressure. Normally, we tested 2 different agents at the same location in the PN to determine the specificity of their effects. In addition, we then moved the stimulating electrode to a different location within the area of the PPN to monitor the effects upon different forms of pacemaker modulations.

By on-line use of a PDP 11/73 computer, we monitored and plotted the instantaneous frequency of the pacemaker signal recorded from the tip of each fish's tail. A simultaneous tape recording of this signal and of the schedules of stimulations and pressure injections served for later analysis of these data.

We performed experiments on 32 individuals of Hypopomus brevirostris, the same species used in our previous study (Kawasaki and Heiligenberg, 1989), and 5 individuals of Eigenmannia lineata. In most inslances, we tested more than 1 drug in 1 fish, often using one as a control for the action of the other. Each drug was tested in at least 3 individuals of Hypopomus and at least 2 individuals of Eigenmannia.

\section{Results}

An overview of pacemaker modulations in gymnotiforms

The PN of Hypopomus consists of electrotonically coupled pacemaker cells, which generate the discharge cycle, and relay cells, which are driven by the pacemaker cells and innervate the spinal motor neurons of the electric organ (Bennett et al., 1967; Bennett, 1971). Whereas the pacemaker cells and their extensive dendrites occupy the dorsal portion of the nucleus, the relay cells fill its ventral portion (Fig. 1, left). As described earlier (Kawasaki and Heiligenberg, 1989), various modulations of the EOD frequency are observed in the context of courtship and can be elicited by stimulating distinct sites in the PPN. Frequency rises are caused by acceleration of the pacemaker cells. They are shown not only in the context of courtship, but also in response to novelties in sensory inputs of any modality. Frequency drops, which can lead to a gradual interruption of EODs, are caused by deceleration of the pacemaker cells. In addition to interruptions following frequency drops, we also observed sudden interruptions which, however, are caused by sustained depolarization of the relay cells and hardly affect the cycle of the pacemaker cells. Chirps are sudden, brief accelerations of the EOD cycle that, in their stronger form, lead to a progressive attenuation of the EOD amplitude. Therefore, chirps are also referred to as "decrement bursts" (Hagedorn, 1983). Chirps are generated by selective recruitment of the relay cells and interfere little with the pacemaker-cell cycle.

In the context of its jamming avoidance response (JAR), $E i$ genmannia gradually raises its EOD frequency in response to an interfering signal of slightly lower frequency and lowers its EOD frequency in response to a signal of a slightly higher frequency. Gradual rises in frequency are caused by enhanced ac- tivity of the small cells in the PPN (Kawasaki et al., 1988) and are reversibly blocked by application of the NMDA receptor blocker APV to the PN (Dye et al., 1989). Gradual decrements in EOD frequency appear to be caused by tonic inhibition of the small cells in the PPN (Rose et al., 1988; Keller and Heiligenberg, 1989). In the context of aggression and courtship, Eigenmannia may abruptly and briefly raise its EOD frequency for several cycles, and a sufficiently strong rise in frequency may lead to a short cessation of the EOD. This behavior, called chirping ("interruptions" by Hopkins, 1974) is triggered by the large cells of the PPN (Kawasaki et al., 1988) and can reversibly be blocked by the kainate/quisqualate receptor blockers GAMS (Dye et al., 1989) and CNQX (present study).

\section{Iontophoresis of glutamate and GABA in the PN}

Glutamate and GABA were iontophoresed in the PN without stimulating the PPN to explore their modulatory effects upon the pacemaker cycle.

In Hypopomus, glutamate and GABA were alternately iontophoresed through the first and second barrel of a triple-barrel microcapillary, while the field potential of the nucleus was recorded through the third barrel containing $\mathrm{NaCl}(3 \mathrm{M})$. The depth of the electrode tip within the PN was estimated by the wave form of the field potential (Fig. 1, right). The first negative peak of the field potential, which reflects the activity of the pacemaker cells, is more prominent at the dorsal locations in the PN. The second negative peak, which reflects the activity of the relay cells, is larger at deeper locations in the PN (Kawasaki and Heiligenberg, 1989). In some penetrations, the first negative peak was further divided into 2 subcomponents. In the case shown in Figure 1, the second negative peak was larger than normal throughout the nucleus, probably reflecting individual variation.

Iontophoresis of glutamate $(100 \mathrm{~mm},-50 \mathrm{nA}, 3 \mathrm{sec})$ induced frequency rises in the EOD most strongly when applied to the dorsal part of the PN, where the first peak of the field potential was maximal. Iontophoresis of GABA $(100 \mathrm{mM},+50 \mathrm{nA})$ induced frequency falls when applied to the dorsal part of the nucleus, though the most sensitive location was located slightly more dorsal than the location giving strongest responses to glutamate (Fig. 1, right). At the most effective site, GABA caused a complete interruption of the EOD.

At slightly deeper locations, sudden frequency modulations that were indistinguishable from naturally occurring chirps could be induced by glutamate, provided that a much stronger iontophoretic current ( $-200 \mathrm{nA}$ ) was used (Fig. 2A). Chirps are generated by synchronized firing of relay cells (Kawasaki and Heiligenberg, 1989). The induction of chirps by steady iontophoresis of glutamate in the PN implies that no synchronized input from the PPN is required for the synchronization of relay cells. At even deeper locations, sudden interruptions could be induced by glutamate, but again, only with a large iontophoretic current ( $-200 \mathrm{nA}$; Fig. $2 B)$. These modulations, induced by the direct application of glutamate to the PN, were indistinguishable from those induced by the stimulation of the PPN (Kawasaki and Heiligenberg, 1989, their figs. 7, 10). While frequency rises could readily be induced with weak iontophoretic currents, chirps and sudden interruptions could not be induced reliably even with strong currents.

In Eigenmannia, iontophoresis of glutamate $(100 \mathrm{~mm},-200$ $\mathrm{nA}, 20 \mathrm{sec}$ ) into the PN induced minor rises in EOD frequency (1-2 Hz). Prolonged iontophoresis failed to induce a further 
A

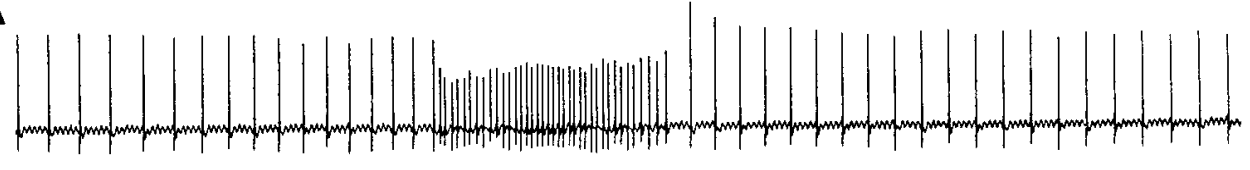

B

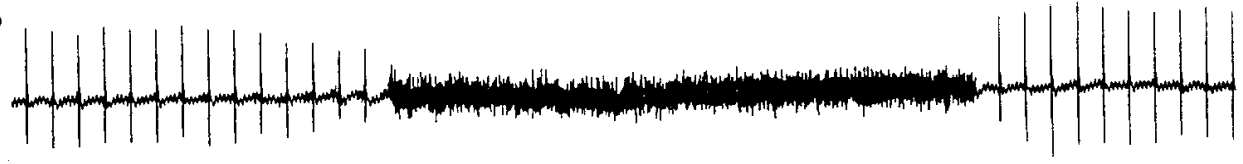

increment in frequency. The elevated frequency rapidly returned to the resting level within several seconds after the iontophoretic current was turned off. When a much larger current was applied $(-2 \mu \mathrm{A}, 10 \mathrm{sec})$, EODs were interrupted after a rapid frequency rise $(10 \mathrm{~Hz})$. Glutamate was effective throughout the nuclcus, though the effects were strongest where the field potential, which in this genus consists of a single peak, was largest. GABA (100 $\mathrm{mM},+2 \mu \mathrm{A}, 30 \mathrm{sec}$ ) was iontophoresed in all parts of the $\mathrm{PN}$, but there was no detectable effect at all.

\section{APV blocks EOD frequency rises in Hypopomus and}

\section{Eigenmannia}

The existence of natural excitation by glutamate in the PN of Hypopomus was tested by injecting pharmacological blockers into the PN by pressure, while stimulating corresponding sites in the PPN to induce behaviors. APV was injected in the PN
Figure 2. A, A chirp (decrement burst) was induced by iontophoresis of glutamate $(100 \mathrm{~mm})$ in the pacemaker nucleus of Hypopomus. The iontophoretic current $(-200 \mathrm{nA})$ was applied over the period indicated by the bar underneath the record. $B$, A sudden interruption was induced by iontophoresis of glutamate to the PN. The iontophoretic current $(-200 \mathrm{nA})$ was started $8 \mathrm{sec}$ before the beginning of the trace and continued over the period indicated by the $h a r$. of Hypopomus, while frequency rises were induced by stimulation of a site in the PPN. As shown in Figure 3, top, APV $(500 \mu \mathrm{M})$ reversibly blocked frequency rises. The blocking effect of APV was observed in 17 nuclei at 33 injection sites. Control injection of artificial cerebrospinal fluid had no effect. At low concentrations of APV (50-500 $\mu \mathrm{M})$, injections at the dorsal part of the PN appeared more effective than injections at the ventral portion. Frequency rises induced by presenting novelties in sensory stimuli were also reversibly blocked by injection of APV (not shown). In agreement with our earlier studies (Dye et al., 1989), APV also blocked EOD frequency rises associated with the JAR in Eigenmannia and lowered the resting frequency of the EOD (Fig. 4, left). As previously suggested (Rose et al., 1988), PPN neurons that control the JAR are spontaneously active, tonically excite the PN, and maintain its resting frequency. Further excitation of the PPN neurons raises the pace-
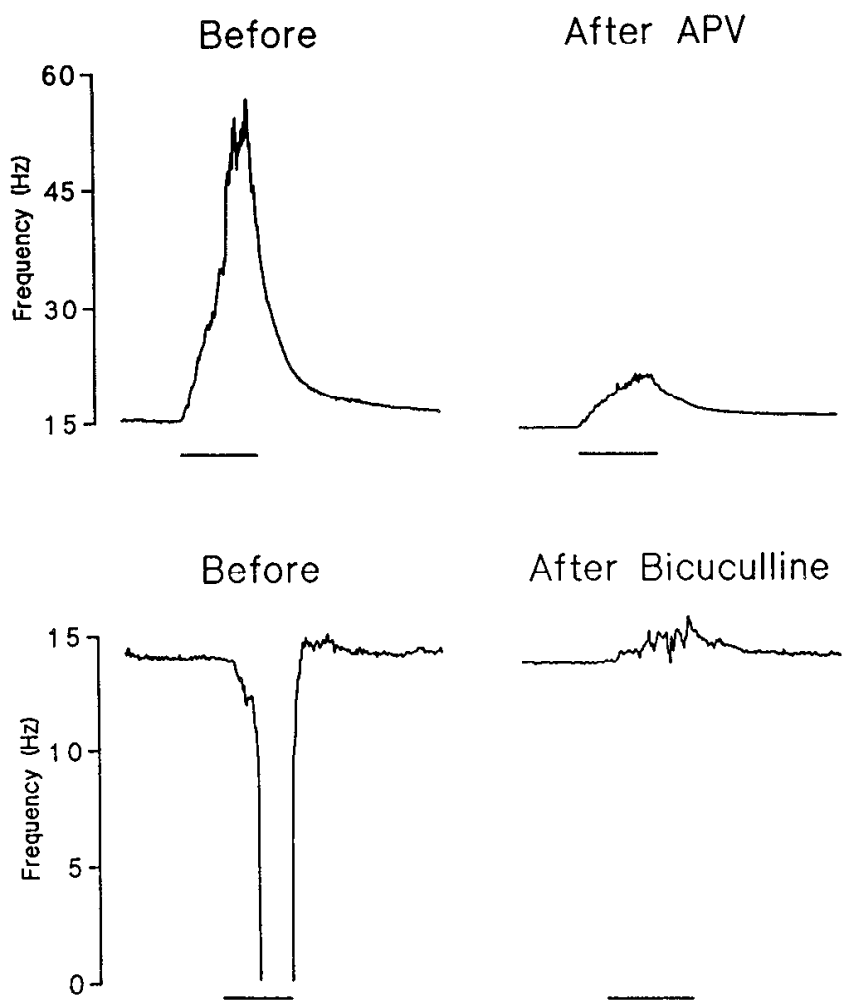

After Bicuculline

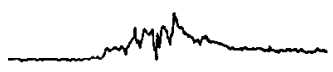

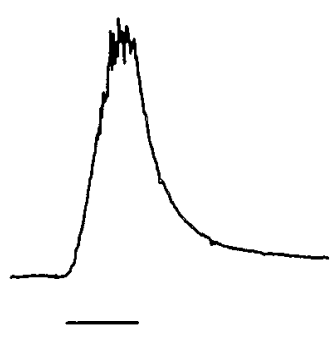

\section{Recovery}

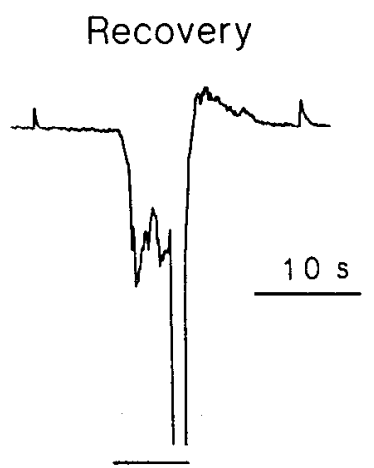

Figure 3. Pacemaker frequency rises (top) and frequency drops (bottom) can be induced selectively by stimulating different sites of PPN in Hypopomus. While application of APV to the PN blocks frequency rises, application of bicuculline blocks frequency drops. Bars underneath the frequency records indicate the period of iontophoretic stimulation of the PPN with L-glutamate. The concentrations of APV and bicuculline were 500 and $200 \mu \mathrm{M}$, respectively. In the upper row, the record in the center was taken $20 \mathrm{sec}$ after pressure injection of APV, and recovery was tested $110 \mathrm{sec}$ later. In the lower row the record in the center was taken 15 sec after pressure injection of bicuculline, and recovery was tested $261 \mathrm{sec}$ later. The small rise in frequency after the injection of bicuculline is due to a weak, simultaneous stimulation of a neighboring site in the PPN causing frequency rises. This response is normally masked by a much stronger frequency drop but, as a result of its longer time course, often appears as the pacemaker recovers from the frequency drop. 
Figure 4. While application of $A P V$ $(500 \mu \mathrm{M})$ to PN of Eigenmannia lowers resting level of pacemaker frequency and suppresses rises in frequency in context of JAR, application of bicuculline ( $2 \mathrm{~mm}$ ) has no effect in this genus. The transient rise in response to the injection of bicuculline was not due to the effect of bicuculline because similar rises were observed when a control solution (artificial cerebrospinal fluid) was injected. The bottom trace indicates the sign of the frequency difference, $D f$, between a jamming signal and the fish's EOD substitute, with positive Dfs causing a drop in pacemaker frequency and negative Dfs causing a rise. Dashed lines indicate absence of the jamming signal to assess the resting frequency of the pacemaker.

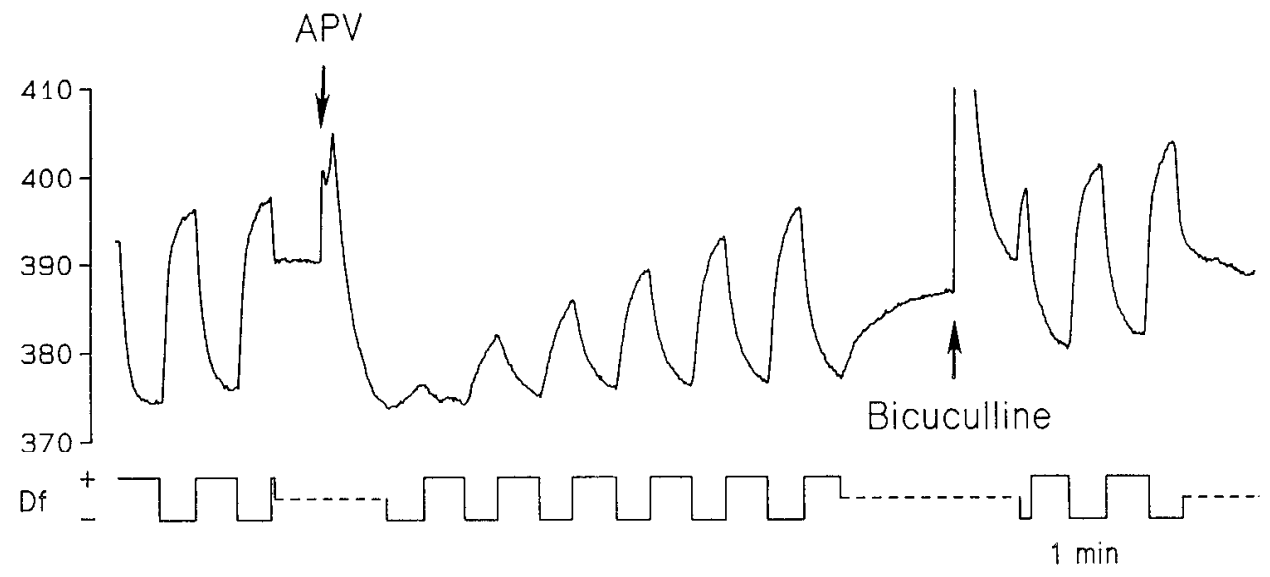

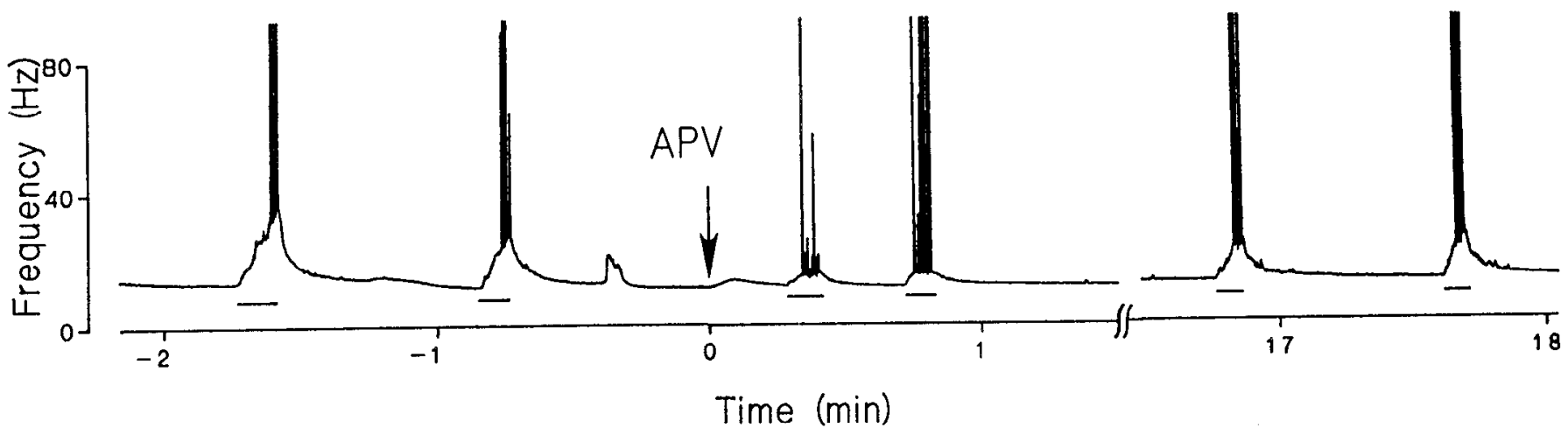

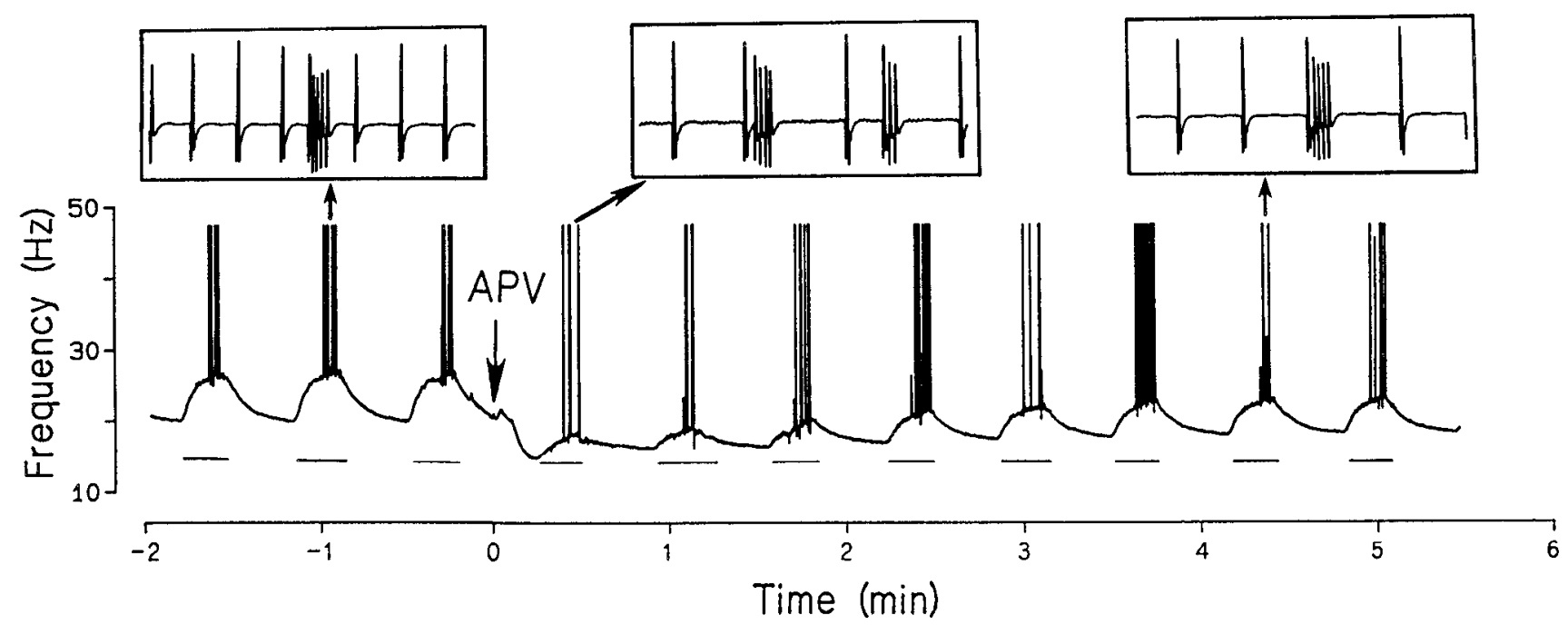

Figure 5. Following injection of $A P V$ into PN of Hypopomus, frequency rises are greatly attenuated, whereas chirping is little affected. The upper and lower graphs represent experiments conducted on different individuals. In both cases, a site in the PPN was chosen that, upon iontophoresis of L-glutamate (bars underneath frequency records), induced frequency rises as well as chirping in the pacemaker. The concentrations of APV were 5 and $60 \mu \mathrm{M}$ in the upper and lower records, respectively. In the case of the lower record, injection of APV lowered the resting frequency of the EOD, as well. This appears to be due to blocking of tonic inputs from the PPN that had been elevated by a long series of glutamate stimulation. Boxes show expanded records of individual chirps embedded in the regular rhythm of EODs. Although chirping seems to occur less frequently immediately following the APV application, its form is not affected by this treatment. Time span of each box is $270 \mathrm{msec}$. 


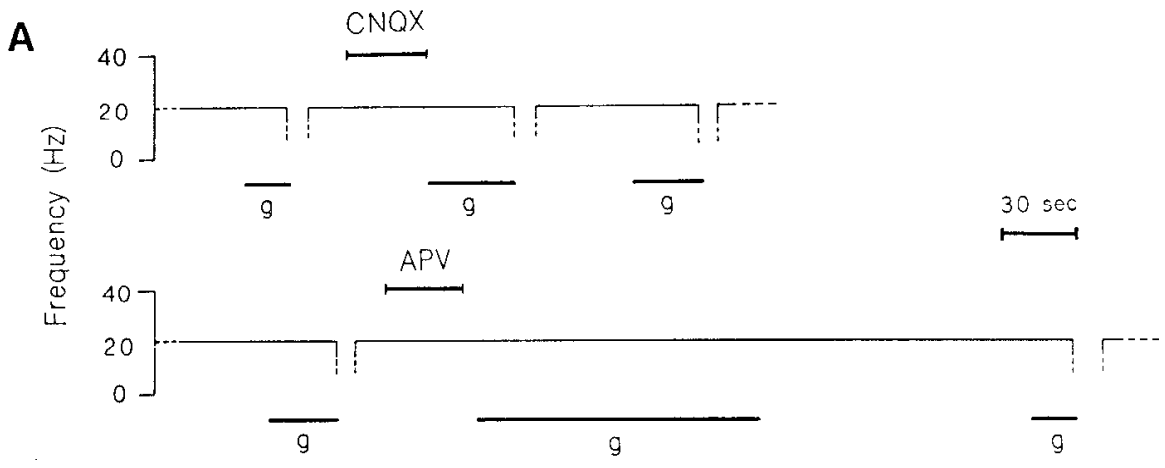

B

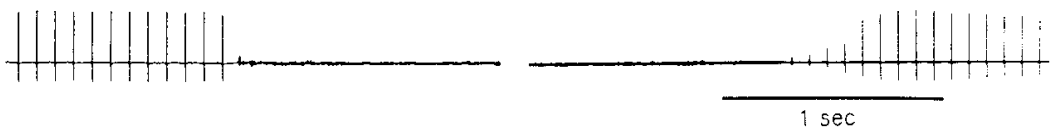

Figure 6. In Hypopomus, $A P V$ also suppresses interruptions of EOD caused by sustained depolarization of its relay cells. Interruptions of this kind, shown as breaks in the EOD frequency records in $A$, can be elicited by iontophoresis of L-glutamate $(\mathrm{g})$ in the vicinity of the PPN (bars underlining records in $A$ ). In contrast, application of $C N Q X$ to the pacemaker has no effect upon interruptions. The left and right traces in $B$ represent the beginning and the end of an interruption that lasted approximately 5 sec. The absence of EOD pulses is marked by elevated noise caused by desynchronized ringing of relay cells. maker frequency, whereas inhibition of the spontaneous activity lowers the pacemaker frequency. Accordingly, after the injection of APV, the resting frequency fell and remained at a low level, and the magnitude of the JAR was diminished. Resting frequency and magnitude of JAR then recovered over the course of several minutes. Control injection of cerebrospinal fluid had no effect on either resting frequency or frequency rises, except for the transient increase of the EOD frequency probably due to mechanical disturbance of the nucleus.

In Hypopomus, a stimulation site in the PPN could be chosen such that frequency rises and chirps were elicited simultaneously. Injection of APV (50-500 $\mu \mathrm{M})$ attenuated frequency rises without significant impairment of chirping. The selective blocking of frequency rises by APV was confirmed in 4 nuclei at 8 injection sites. At higher concentration ( $5 \mathrm{~mm}$ ), however, APV also suppressed chirping to a minor extent (Fig. 5). This agrees with a similar selectivity in the action of APV reported earlier for Eigenmannia (Dye et al., 1989). The threshold concentration of APV, necessary for a significant attenuation of frequency rises in Hypopomus, was $50 \mu \mathrm{M}$.

\section{APV blocks the sustained depolarization of relay cells in Hypopomus}

Hypopomus can suddenly silence its EOD by sustained and selective depolarization of its relay cells, and this response can be elicited by stimulating various locations in the caudal PPN (Kawasaki and Heiligenberg, 1989). APV $(50 \mu \mathrm{M})$ blocked this response reversibly (Fig. 6). The blocking effects were observed with 7 injections made in 6 nuclei. It was not clear whether the injection into the ventral part of the PN was more effective. Injection of either bicuculline ( $2 \mathrm{mM}$ ), CNQX ( $500 \mathrm{~mm}$ ), or the control solution had no effect on this type of interruption.

CNQX selectively blocks chirping in Hypopomus and Eigenmannia

CNQX $(500 \mu \mathrm{M})$ was injected in the PN of Hypopomus while gradual rises and chirps were induced simultaneously through stimulation of the PPN. While its effect upon gradual frequency rises was minimal, chirping was significantly and reversibly suppressed (Fig. 7). Applications of CNQX appeared to be most effective at the deeper location in the nucleus. We tested APV and CNQX at the same injection site within the PN by using separate barrels of the triple-barrel electrode. By alternately injecting APV and CNQX at concentrations of 50 and $500 \mu \mathrm{M}$, respectively, we could demonstrate the selective effects of these 2 agents. For example, the data in the lower half of Figure 5 and in Figure 7 were obtained with consecutive injections at the same location. This selectivity persisted through successive applications of the 2 drugs over the course of hours. We observed that CNQX selectively blocked chirps but not frequency rises in 12 cases. Injection of the control solution had no effect on chirping.

We also applied CNQX to the PN of Eigenmannia and found that it selectively suppressed chirping, without affecting the JAR. This supports our earlier conclusion (Dye et al., 1989), based on the application of GAMS, that chirping is mediated by a kainate/quisqualate receptor.

\section{Iontophoresis of NMDA, kainate, and quisqualate in the $P N$}

NMDA, kainate, and quisqualate were iontophoresed into the PN without stimulating the PPN to assess their direct effects upon the pacemaker cycle.

In IIypopomus, NMDA (2-5 mM), kainatc (2-10 mM), and quisqualate $(10 \mathrm{mM})$ were iontophoresed at different depths in the pacemaker using the same method as used for the glutamate iontophoresis. All these drugs strongly induced frequency rises at the dorsal part of the nucleus. These drugs were 10-50 times more potent than glutamate: A concentration of $2-10 \mathrm{~mm}$, applied with $-50 \mathrm{nA}$, evoked frequency rises of the EOD that were stronger and longer lasting than those evoked by $100 \mathrm{~mm}$ glutamate and with the same amount of current. The long-lasting aftereffects are probably due to the lack or the weakness of natural uptake mechanisms for these agonists. At an injection site near the center of the nucleus, where NMDA induced only smooth frequency rises, kainate induced a chirplike jitter in the EOD pattern, in addition to smooth frequency rises (Fig. 8). Sudden interruptions, which are characterized by desynchronized EODs, could be induced by iontophoresis of NMDA at decper locations in the nucleus. The onset, offset, and quality of the desynchronized EOD trace during the interruption were 


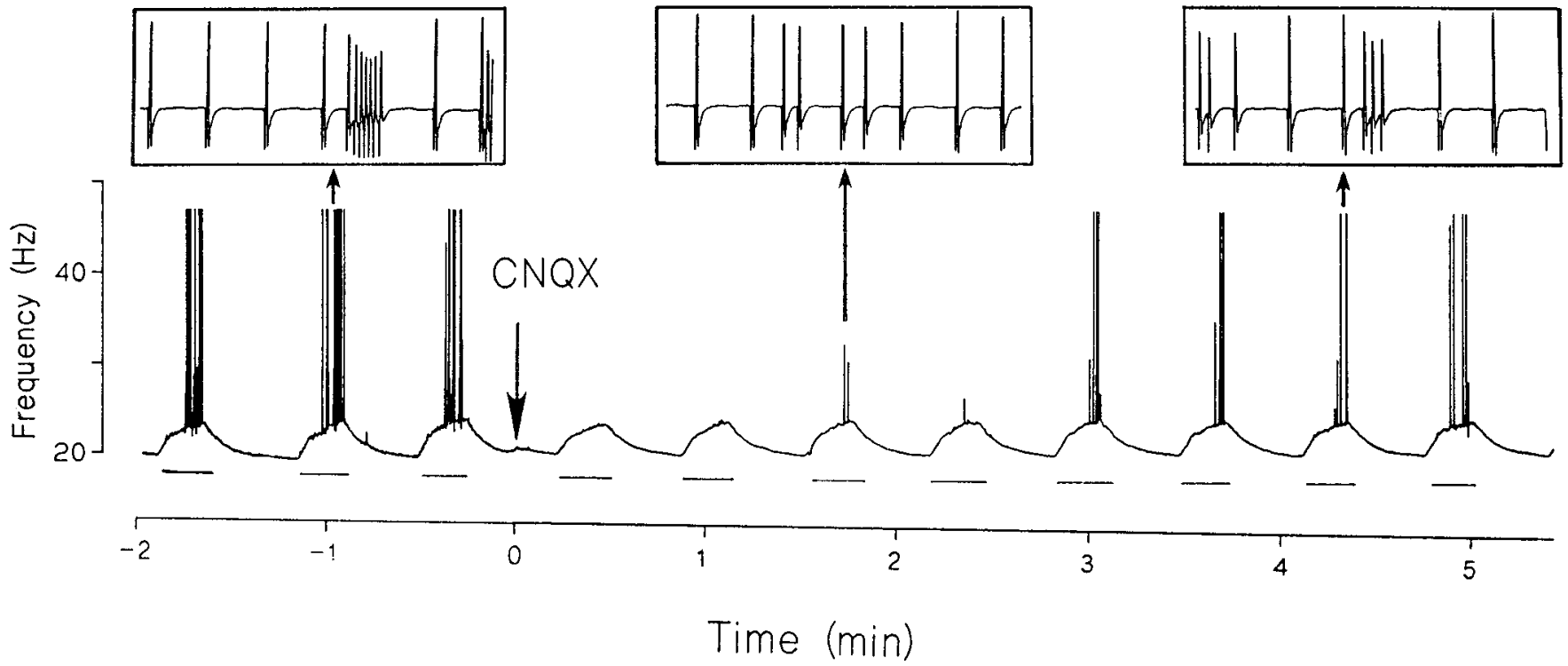

Figure 7. Following injection of $\operatorname{CNQX}(250 \mu \mathrm{M})$ to PN of Hypopomus, chirping is largely suppressed, whereas frequency rises are not affected. As in Figure 5, a site in the PPN was chosen that induced chirping as well as frequency rises in response to L-glutamate (bars underneath frequency record). This record was taken $8 \mathrm{~min}$ after the lower record in Figure 5 in the same fish, with the triple-barrel pipettes remaining at the same location within the pacemaker, demonstrating that the selectivity of the action of the 2 drugs is not an artifact of the application site. The expanded traces (boxes) of individual chirps from the longer record underneath show that the intensity of chirping, that is, the rate of pulses within a burst, is also reduced by CNQX. Time span of each box is $270 \mathrm{msec}$.

indistinguishable from those in the interruptions presented in Figure $2 B$. Although less effective than alternately iontophoresed NMDA ( $2 \mathrm{mM},-50 \mathrm{nA})$, kainate $(2 \mathrm{mM},-50 \mathrm{nA})$ and quisqualate $(10 \mathrm{~mm},-50 \mathrm{nA})$ could also induce sudden interruptions in some penetration. As was the case with glutamate iontophoreses, chirps and sudden interruptions were difficult to induce consistently by iontophoresis of NMDA, kainate, or quisqualate.

In Eigenmannia, iontophoresis of NMDA $(10 \mathrm{mM},-60 \mathrm{nA})$ caused gradual acceleration in EOD frequency. As long as the iontophoresis was continued (tested up to $80 \mathrm{sec}$ ), the EOD frequency was gradually increased to $50 \mathrm{~Hz}$ above the resting frequency. The recovery was very slow: It took more than 100 sec to return to the resting level after the current was turned off. Kainate ( $5 \mathrm{~mm},-30 \mathrm{nA}$ ) iontophoresed at the same location caused a short-term acceleration and a following interruption of the EOD: During the first $10 \mathrm{sec}$ of iontophoresis, the EOD frequency increased to $20 \mathrm{~Hz}$ above the resting frequency; then, the frequency rapidly fell, and the FODs were interrupted. The EODs resumed about $30 \mathrm{sec}$ after the current was turned off. Quisqualate ( $5 \mathrm{~mm},-30 \mathrm{nA}$ ) had exactly the same effects. Such differential effects of NMDA and kainate/quisqualate were seen over a depth of $350 \mu \mathrm{m}$.

\section{GABA inhibits the pacemaker cycle in Hypopomus but not in Eigenmannia}

The existence of physiological inhibition by GABA in the PN of Hypopomus was tested by injecting bicuculline into the PN by pressure, while stimulating an appropriate site in the PPN to induce frequency falls and interruptions. As shown in the lower half of Figure 3, bicuculline $(200 \mu \mathrm{M})$ reversibly blocked frequency falls and interruptions. Control solution of artificial cerebrospinal fluid injected through a neighboring barrel had no effect. The blocking effects of bicuculline were observed in 6 nuclei with 12 injections. Bicuculline appeared most effective when applied to the dorsal part of the PN, suggesting that GABAergic inhibition acts upon the pacemaker cells (Kawasaki and Heiligenberg, 1989). Injection of APV (5 mM) had no effect on frequency falls and subsequent interruptions in Hypopomus.

Injections of bicuculline ( $2 \mathrm{~mm}$ ) were made at 7 different sites within the PN of an Eigenmannia. Rises and falls of the EOD frequency observed during the JAR of Eigenmannia were not affected at any injection site (Fig. 4, right), supporting our earlier interpretation that this genus maintains its resting EOD frequency by tonic activity of the small cells in the PPN and lowers its EOD frequency by tonic inhibition of these cells. In 2 other
Figure 8. NMDA (5 mM) and kainate $(K A ; 10 \mathrm{~mm})$ were alternately iontophoresed at same location in PN of Hypopomus. While NMDA induced smooth frequency rises, kainate induced a strong jitter in the timing of the EODs. The wave form of the field potential recorded at this location was similar to the one in the fourth row from the bottom in Figure 1, right.

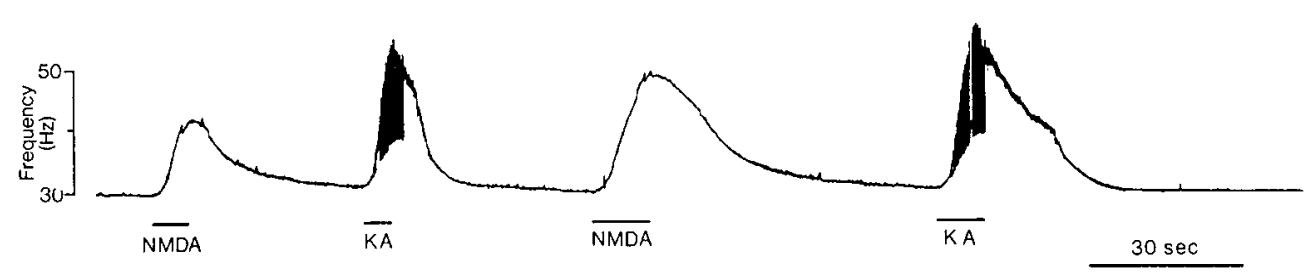




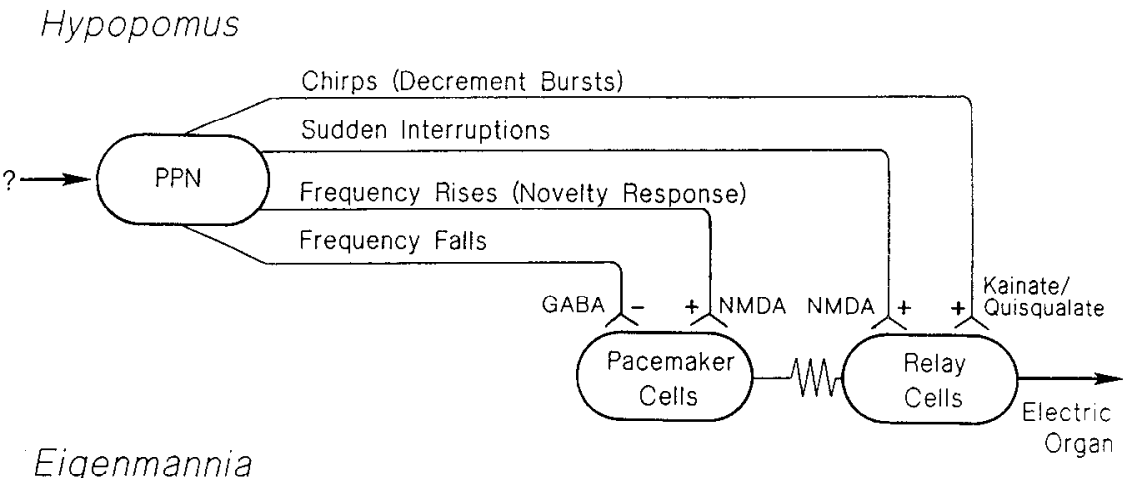

Eigenmannia

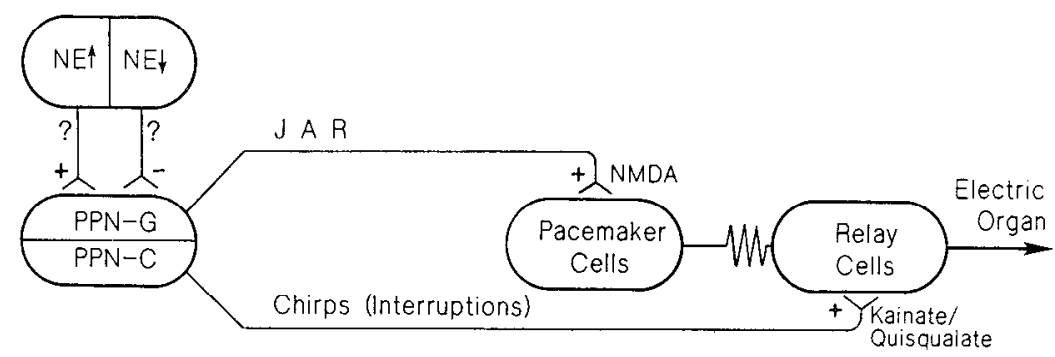

Figure 9. Prepacemaker-pacemaker organization in Hypopomus and Eigenmannia. In Eigenmannia, the $P P N$ is subdivided into $P P N-G$ and $P P N-C$, which, when stimulated, cause frequency rises and chirps, respectively (Kawasaki et al., 1988). $N E \uparrow$ and $N E \downarrow$ are subdivisions in the nucleus electrosensorius that, respectively, cause frequency rises and frequency falls when stimulated (Keller and Heiligenberg, 1989). Substructures of and inputs to the prepacemaker nucleus of Hypopomus are unknown. nuclei, bicuculline and APV were alternately injected through different barrels of an electrode. Again, bicuculline had no effect, whilc APV blocked frequency rises.

\section{Discussion}

This study demonstrates that 2 genera of gymnotiform electric fish, Hypopomus and Eigenmannia, appear to exploit the kinetically distinct properties of different glutamate receptors to generate different forms of pacemaker accelerations, whereas they differ in their means of decelerating the pacemaker cycle. The specific and selective blocking effects of APV and CNQX indicate that NMDA receptors mediate gradual accelerations of pacemaker cells and the sustained depolarization of relay cells, whereas kainate/quisqualate receptors mediate the brief and rapid modulations, or chirping, of relay cell firing. This observation is in agreement with studies in other systems, such as the spinal cords of lampreys (Grillner et al., 1987) and larval Xenopus (Roberts et al., 1986) or the X cells in the mammalian lateral geniculate (Heggelund and Hartveit, 1989), demonstrating that, as a likely consequence of their differences in kinetics, NMDA receptors mediate slow and sustained processes, while kainate/quisqualate receptors mediate fast and transient processes.

Figure 9 represents our current hypothesis of the prepacemaker-pacemaker organization in Hypopomus and Eigenmannia. In both genera, gradual rises in pacemaker frequency appear to be generated through effects on the pacemaker cells, whereas chirping is brought about by selective recruitment of the relay cells (Dye, 1988; Dye et al., 1989; Kawasaki and Heiligenberg, 1989). In Eigenmannia, we assume that different input fibers from the PPN induce frequency rises and chirps, respectively, by terminating onto NMDA- and kainate/quisqualate-sensitive postsynaptic membranes of different cell types. An additional complexity emerges in Hypopomus, however, which has the behavioral option of selectively silencing its relay cells through sustained depolarization (Kawasaki and Heiligenberg, 1989).
Because this form of EOD interruption is blocked by APV, this behavior appears to be mediated by NMDA receptors. The relay cells of $H_{y}$ popomus thus are subject to the dual control by NMDA and kainate/quisqualate receptors. It remains to be determined whether the NMDA receptors mediating sudden interruptions reside at locations different from those of the kainate/quisqualate receptors mediating chirping. The inhibitory control over the PN demonstrated in Hypopomus represents an additional complexity of the prepacemaker-pacemaker organization in this genus.

By separate modulatory innervations of pacemaker cells and relay cells, gymnotiform fish are able to alter the firing of their $\mathrm{PN}$ in different ways and to generate a variety of distinct social signals. The independence of the control of pacemaker cells and relay cells in Hypopomus becomes most obvious when a stimulation site in the PPN triggers both silencing of the pacemaker cells and chirping of the relay cells. In this instance, chirps appear in the absence of normal EOD activity. Although, in the normally behaving animal, chirping occurs in conjunction with a gradual rise in EOD frequency, this observation demonstrates that such slow rises are not required for chirping.

While chirping, the relay cells of Hypopomus fire in synchrony, at a rhythm much faster than that of the pacemaker cells (Kawasaki and Heiligenberg, 1989). Because chirping can be elicited directly by glutamate injection to the PN, the synchronization of the relay cells does not require the control of the PPN and therefore must be based upon local, yet unknown network properties.

\section{References}

Bennett MVL (1971) Electric organs. In: Fish physiology, Vol 5 (Hoar WS, Randall DJ, eds) pp 493-574. New York: Academic.

Bennett MVL, Pappas GD, Gimenez M, Nakajima Y (1967) Physiology and ultrastructure of electrotonic junctions. IV. Medullary electromotor nuclei in gymnotiform fish. J Neurophysiol 30:236-300.

Dye J (1988) An in vitro physiological preparation of a vertebrate 
communicatory behavior: chirping in the weakly electric fish, Apteronotus. J Comp Physiol A 163:445-458.

Dye J, Heiligenberg W, Keller CH, Kawasaki M (1989) Different classes of glutamate receptors mediate distinct behaviors in a single brainstem nucleus. Proc Natl Acad Sci USA 86:8993-8997.

Getting P (1989) Emerging principles governing the operation of neural networks. Annu Rev Neurosci 12:185-204.

Grillner S, Wallén P, Dale N, Brodin L, Buchanan J, Hill R (1987) Transmitters, membrane properties and network circuitry in the control of locomotion in lamprey. Trends Neurosci 10:34-40.

Hagedorn M (1983) Social signals in electric fish. PhD thesis. University of California, San Diego.

Hagedorn M (1986) The ecology, courtship, and mating of gymnotiform electric fish. In: Electroreception (Bullock T, Heiligenberg W, eds) pp 497-525. New York: Wiley.

Harris-Warrick RM (1988) Chemical modulation of central pattern generators. In: Neural control of rhythmic movements (Cohen $\mathrm{AH}$, Rossignol S, Grillner S, eds) pp 285-331. New York: Wiley.

Harris-Warrick RM, Marder EE (1991) Modulation of neural networks for behavior Annu Rev Neurosci 14:39-57.

Heggelund P, Hartveit H (1989) Lagged and non-lagged X-cells in the cat lateral geniculate nucleus receive retinal input through different glutamate receptors. Soc Neurosci Abstr 75:5.

Heiligenberg W, Bastian J (1980) Species specificity of electric organ discharges in sympatric gymnotoid fish of the Rio Negro. Acta Biol Venezuela 10:187-203.

Hopkins CD (1974) Electric communication: functions in the social behaviors of Eigenmannia virescens. Behavior 50:270-305.

Hopkins CD, Heiligenberg W (1978) Evolutionary designs for electric signals and electroreceptors in gymnotoid fishes of Surinam. Behav Ecol Sociobiol 3:113-134.

Hopkins CD, Westby GWM (1986) Time-domain processing of electric organ discharge waveforms by pulse-type electric fish. Brain Behav Evol 29:77-104.

Kawasaki M, Heiligenberg W (1989) Distinct mechanisms of modulation in a neuronal oscillator generate different social signals in the electric fish Hypopomus. J Comp Physiol A 165:731-741.

Kawasaki M, Maler L, Rose G., Heiligenberg W (1988) Anatomical and functional organization of the prepacemaker nucleus in gymnotiform electric fish: the accommodation of two behaviors in one nucleus. J Comp Neurol 276:113-131.

Keller CH, Heiligenberg W (1989) From distributed sensory processing to discrete motor representations in the diencephalon of the electric fish, Eigenmannia. J Comp Physiol A 164:565-576.

Marder EE (1987) Neurotransmitters and neuromodulators. In: The crustacean stomatogastric system (Selverston AI, Moulins M, eds) pp 263-300. Berlin: Springer.

Roberts A, Soffe SR, Dale N (1986) Spinal interneurons and swimming in frog embryos. In: Neurobiology of vertebrate locomotion (Grillner S, Stein PSG, Stuart DG, Forssberg H, Herman RM, eds) pp 279306. Basingstoke, UK: Macmillan.

Rose GJ, Kawasaki M, Heiligenberg W (1988) 'Recognition units' at the top of sensory hierarchy? Prepacemaker neurons in Eigenmannia code the sign of frequency differences unambiguously. J Comp Physiol A $162: 759-772$.

Westby GWM (1975) Comparative studies of the aggressive behaviors of two gymnotid electric fish (Gymnotus carapo and Hypopomus artedi). Behav Ecol Sociobiol 4:381-393. 